\title{
The bound polaron with weak electron-lattice coupling
}

\begin{abstract}
The binding energy of an electron to a Coulomb potential is evaluated in the limit of weak coupling to optic modes. The results are compared with bounds given by other workers.
\end{abstract}

The properties of trapped electrons are sensitive to the electron-lattice interaction. In polar materials this coupling is dominated by the interaction with longitudinal optic modes. Even in the limit of weak coupling, and with the simplifying assumptions that all optic modes have the same frequency and that the electron is bound by a pure Coulombic potential, it does not seem possible to obtain an exact analytic expression for the change in ground-state energy caused by the interaction. The only published expressions (Platzman 1962, Larsen 1969) are too complicated even for numerical treatment, and only weak bounds have been derived. We show here that the expressions for the change in ground-state energy can be rewritten in a form which can be evaluated numerically, and we compare the results with other expressions.

In the absence of electron-lattice interaction the electron binding energy is just $R$, the appropriate Rydberg which uses the band mass and includes electronic screening through the optic dielectric constant. The optic phonons are assumed to have a single frequency, $\omega_{0}$. The electron-phonon interaction is assumed weak, so that the dimensionless constant $\alpha$, which measures its strength, is small; Pines (1964) $\dagger$ suggests $0 \leqslant \alpha \lesssim \frac{1}{2}$ in the corresponding free polaron problem.

The lowest-order correction to the ground-state energy can be written (Platzman 1962)

$$
\delta^{\prime}=-\sum_{k} \sum_{n}\left(\hbar \omega_{0}\right)^{2}\left|V_{k}\right|^{2} \frac{|\langle n|\exp (-\mathrm{i} \boldsymbol{k} . \boldsymbol{r})| 0\rangle|^{2}}{E_{n}-E_{0}+\hbar \omega_{0}}
$$

where $V_{k}$ is defined by Pines in terms of $k, \alpha$ and $\omega_{0} . E_{n}$ and $|n\rangle$ are the electronic eigen values and eigenstates (including continuum contributions) in the absence of coupling.

We begin by eliminating the sum over intermediate states. In doing this we introduce $\mathscr{H}_{0} \equiv p^{2} / 2 m^{*}+V(r)$, the Hamiltonian in the absence of coupling. The part of (1) needed is

$$
\begin{aligned}
S & =\sum_{n} \frac{|\langle n|\exp (-\mathrm{i} \boldsymbol{k} \cdot \boldsymbol{r})| 0\rangle| 2}{E_{n}-E_{0}+\hbar \omega_{0}} \\
& =\sum_{n}\langle 0|\exp (-\mathrm{i} \boldsymbol{k} \cdot \boldsymbol{r})| n\rangle\left\langle n\left|\frac{1}{E_{n}-E_{0}+\hbar \omega_{0}} \exp (\mathrm{i} \boldsymbol{k} \cdot \boldsymbol{r})\right| 0\right\rangle \\
& =\sum_{n}\langle 0|\exp (-\mathrm{i} \boldsymbol{k} \cdot \boldsymbol{r})| n\rangle\left\langle n\left|\frac{1}{\mathscr{H}_{0}-E_{0}+\hbar \omega_{0}} \exp (\mathrm{i} \boldsymbol{k} \cdot \boldsymbol{r})\right| 0\right\rangle \\
& =\Sigma\left\langle 0\left|\exp (-\mathrm{i} \boldsymbol{k} \cdot \boldsymbol{r}) \frac{1}{\mathscr{H}_{0}-E_{0}+\hbar \omega_{0}} \exp (\mathrm{i} \boldsymbol{k} \cdot \boldsymbol{r})\right| 0\right\rangle
\end{aligned}
$$

This can be simplified further, taking the exponentials into the denominator and giving

$$
S=\left\langle 0\left|\left\{\exp (-\mathrm{i} \boldsymbol{k}, \boldsymbol{r}) \mathscr{H}_{0} \exp (\mathrm{i} \boldsymbol{k}, \boldsymbol{r})-\mathscr{H}_{0}+\hbar \omega_{0}\right\}^{-1}\right| 0\right\rangle
$$

$\uparrow$ The articles by H. Frölich and T. D. Schutz in this reference are excellent sources of background material. 
or, with our expression for $\mathscr{H}_{0}$,

$$
S=\left\langle 0\left|\left\{\hbar \omega_{0}+\hbar^{2} k^{2} / 2 m^{*}+\hbar \boldsymbol{k} \cdot \boldsymbol{p} / m\right\}^{-1}\right| 0\right\rangle .
$$

This expression is closely analogous to the result for the free polaron given by Pines.

The energy reduction, $\delta^{\prime}$, is a measure of the absolute change in groundstate energy. However, for most purposes of interest we need $\delta$, the change in ionization energy. Consequently we subtract from $\delta^{\prime}$ the energy reduction for a free polaron with zero momentum. This gives

$\delta=-\sum_{\boldsymbol{k}}\left(\hbar \omega_{0}\right)^{2}\left|V_{\boldsymbol{k}}\right|^{2}\left\langle 0\left\{\frac{1}{\hbar \omega_{0}+\left(\hbar^{2} k^{2} / 2 m^{*}\right)+\left(\hbar \boldsymbol{k} \cdot \boldsymbol{p} / m^{*}\right)}-\frac{1}{\hbar \omega_{0}+\left(\hbar^{2} k^{2} / 2 m^{*}\right)}\right\} \mid 0\right\rangle(5)$

Platzman and Larsen obtained bounds on $\delta$ by methods equivalent to expanding the denominator in (5) as a Taylor series in $\left(\hbar k \cdot p / m^{*}\right) /\left(\hbar \omega_{0}+\hbar^{2} k^{2} / 2 m^{*}\right)$. Only a few terms in this expansion converge, so, although adequate for bounds, it is inadequate for more exact solution.

Direct evaluation of (5) is possible after some rearrangement. The sum over $k$ is replaced by an integral (strictly over the first Brillouin zone; in practice over all $\boldsymbol{k}$ space is acceptable)

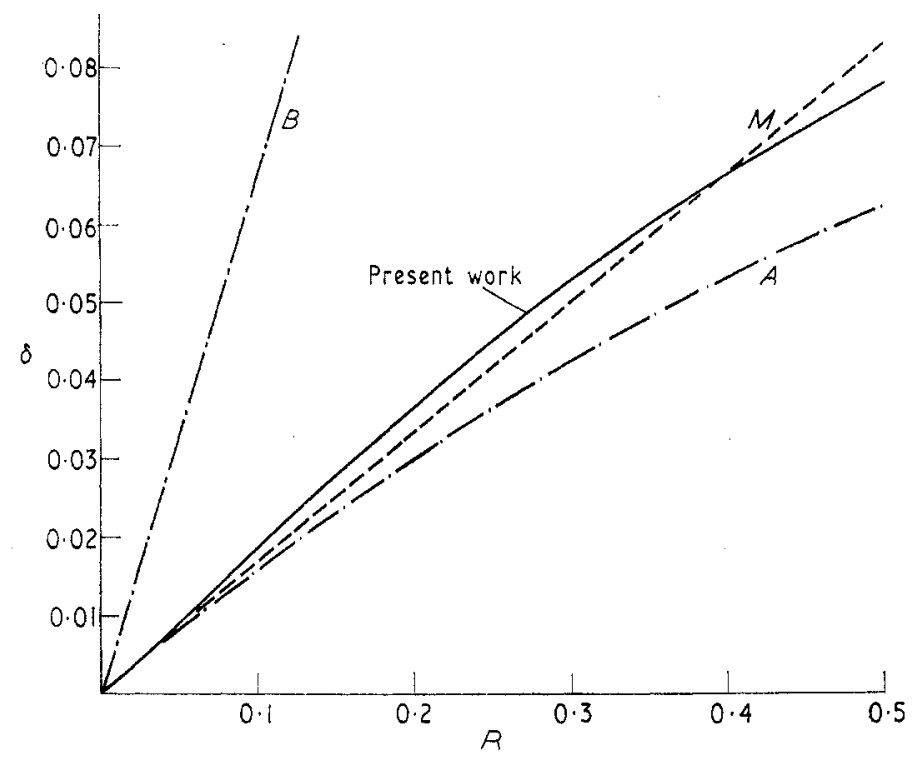

Figure 1. Increase of binding energy $\delta$ as a function of $R$, the unperturbed binding energy. Both energies are in units of $\hbar \omega_{0}$. The bounds are $A \equiv \frac{1}{6}\left(R / \hbar \omega_{0}\right)-\frac{1}{3}\left(R / \hbar \omega_{0}\right)^{2}$ and $B \equiv \frac{2}{3}\left(R / \hbar \omega_{0}\right)$. The approximation that the electron mass alone is changed gives $M \equiv \frac{1}{6}\left(R / \hbar \omega_{0}\right)$.

and we rewrite the matrix element by expanding $|0\rangle$ in momentum eigenfunctions. Many of the integrals can then be carried out analytically. The result is a two-dimensional integral which has a weak (integrable) singularity corresponding to $-\hbar \boldsymbol{k} \cdot \boldsymbol{p} / \mathrm{m}^{*}=\hbar \omega_{0}+\hbar^{2} k^{2} / 2 \mathrm{~m}^{*}$. This integral can be carried out numerically, with due care because of the singularity.

In figure 1 we show $\delta$ as a function of $R / \hbar \omega_{0}$. Also shown are the bounds derived by other workers. It can be seen that the Platzman conjecture, as corrected by Larsen, is only valid 
for very small $R$, typically $R \leqq 0.03 \hbar \omega_{0}$. However, the approximation that the only effect of the coupling is to change the effective mass:

$$
m^{*}>m_{\mathrm{p}}=m^{*}\left(1+\frac{1}{6} \alpha\right)
$$

is always better and is quite accurate, even to large values of $R / \hbar \omega_{0}$.

There do not seem to be any systems for which a direct comparison of theory and experiment is possible. However, these results may give limited guidance for centres in the silver halides where the coupling is relatively weak (Hodby et al. 1967).

Theoretical Physics Division,

A. M. Stoneman

Building 8.9,

22nd May, 1970

Atomic Energy Research Establishment,

Harwell, Didcot,

Berkshire,

England, UK

Hodby, J. W., Borders, J. A., Brown, F. C., and Foner. S., 1967, Phys. Rev. Lett., 19, 952-5.

LARSEN, D. M., 1969, Phys. Rev., 187, 1147-52.

Pines, D., Polarons and Excitons, 1963, Eds. C. G. Kuper and G. D. Whitfield (Edinburgh: Oliver and Boyd) pp. 33-44.

Platzman, P. M., 1962, Phys. Rev., 125, 1961-5.

\title{
The Koster-Slater method for F-centre levels
}

\begin{abstract}
The energy levels of the $F$ centre have been discussed using the KosterSlater approach. It has been shown that a simple two-band model reproduces the deep states of the $F$ centre. The ground state is pulled up from the valence band while the excited states are pulled down from the conduction band.
\end{abstract}

In this letter the feasibility of a Koster-Slater (1954) type of approach for obtaining F-centre levels is discussed. The general theory of this method as applied to defect problems is well known and has been discussed by Bassani et al. (1969). Callaway and Hughes (1967) have also done a very detailed calculation for impurity states associated with a vacancy in silicon using this method.

The energy levels of the $F$ centre electron are given by the zeros of the determinant (Callaway and Hughes 1967):

$$
\operatorname{det}\left|I_{\mathrm{A}}+G_{\mathrm{A}}(E) V_{\mathrm{A}}\right| \quad \text { where } \quad G_{\mathrm{A}}(E)=\left(H_{0}-E\right)_{\mathrm{A}}{ }^{-1}
$$

$H_{0}$ is the Hamiltonian of the perfect periodic crystal and $V_{\mathrm{A}}$ are the Wannier matrix elements of $V$, the change in potential due to the negative ion vacancy. The suffix $A$ indicates that only the defect region is considered. In $\mathrm{NaCl}$, for instance, $V$ could approximately be the negative of the $\mathrm{Cl}^{-}$ion potential which has been given by Abrenkov and Antonova (1967). An inspection of the potential $V$ indicates that at the vacancy site it is strongly positive, and at the nearest neighbour site it is negative. The simplest model to reproduce the F-centre levels would be a two-band model, having the feature that the positive Wannier matrix elements $V(0,0)$ would pull up a level from the valence band and the negative Wannier matrix elements $V(\Delta, \Delta)$, where $\Delta$ is the nearest neighbour vector, would pull down the excited states from the conduction band. 